\title{
Applying genetics to the splicing problem
}

Perhaps as surprising as the presence of introns in protein-coding genes is the complexity of the splicing machinery used to remove introns from the transcripts of those genes. The catalog of components of this machinery now includes several small nuclear (sn) RNAs with their associated proteins, and a variety of other proteins. Many of these components are assembled in the spliceosome (a particle sedimenting at $60 \mathrm{~S}$ ), suggesting a total molecular weight of nearly $3 \times 10^{6}$ (for review, see Green 1986; Padgett et al. 1986). This complexity is all the more surprising because chemically it appears unnecessary: RNA molecules can and do splice themselves (Cech and Bass 1986).

The splicing apparatus has been partially analyzed in vitro by using biochemical or genetic means to deplete the reaction of one or more components, followed by complementation with purified components. Mammalian systems have been used in this way to prove that both U1 and U2 snRNAs are essential for splicing, to separate a crude extract into several fractions that complement each other, and finally to demonstrate that splicing is carried out in a large complex whose formation is dependent on intact splice sites and ATP. This observation is important both for theoretical reasons and for the practical one that it is now potentially possible to purify most of the components of the splicing reaction at one pass (Green 1986; Padgett et al. 1986).

Substantial progress has also been made using Saccharomyces cerevisiae, whose biochemical and genetic attributes make it a useful organism for analyzing this complex process. On the whole, splicing is similar in all eukaryotic organisms. However, Saccharomyces differs in a few details. The acceptor for lariat formation is an invariable-UAUAAC - the third A always carrying the triple phosphodiester bond (Domdey et al. 1984; Rodriquez et al. 1984). The "small" nuclear RNA corresponding to the U2 snRNA of mammalian cells is 1175 nucleotides long, and also contains some sequences that may be homologs to U4, U5, and U6 snRNAs (Ares 1986). Cleavage at the $5^{\prime}$ splice junction and lariat formation require the correct sequence at both sites but not at the 3' splice junction (Rymond and Rosbash 1985). This result suggests that in yeast the fixed sites of intron recognition are the G/GUAUCU at the $5^{\prime}$ junction and the UACUAAC 8-50 nucleotides upstream of the AG/ at the $3^{\prime}$ junction. Indeed, the presence of those sequences has been used to predict the presence of an intron (Miller 1984). On the other hand Saccharomyces does not splice mammalian introns, which have a much more flexible sequence requirement for cleavage and lariat formation.
In most respects the yeast splicing apparatus is similar to that of vertebrates. A $40 S$ splicing complex has been identified whose formation is largely dependent on ATP as well as on an intact $5^{\prime}$ splice site and a lariat acceptor site, but not on a 3' splice site (Brody and Abelson 1985; Pikielny and Rosbash 1986). High-resolution analysis, using gel electrophoresis and oligo(dT) chromatography, reveals that at least two and perhaps three classes of spliceosome are formed in vitro (Pikielny and Rosbash 1986; Pikielny et al. 1986). Spliceosome I contains intact substrate, as well as at least four snRNA molecules: LSR1 (the 1175-nucleotide U2 equivalent), $\operatorname{snR} 7_{L}$ and snR7s (215 and 185 nucleotides, respectively), and snR14 (160 nucleotides). This migrates slightly slower than, and appears to be a precursor to, the spliceosome II, which contains some unspliced substrate as well as the products of the splicing reaction, exon 1 and the lariat structure, but little if any fully spliced substrate; as isolated, it includes no snR14. These complexes are stable in the presence of EDTA and excess competitor RNA, as well as to the rigors of electrophoresis, and therefore may represent only the core of the spliceosome.

The genetics of the snRNAs has been studied extensively by Guthrie and co-workers. They have identified more than two dozen such RNAs (Riedel et al. 1986). Several are not essential for growth (Guthrie et al. 1986), but three are: LSR1, also known as snR20 (Ares 1986), and snR7 and snR14 (B. Patterson and C. Guthrie, pers. comm.). The gene for snR7 gives rise to both $s n R 7_{L}$ and $s n R 7_{s}$. All four of these RNA species are found in the spliceosome (Pikielny et al. 1986).

An important element in the genetic analysis of splicing in yeast is the availability of mutants in several genes involved in mRNA splicing. No one has yet devised a selection scheme for such mutants; these were isolated because of the following fortuitous quirk in the yeast genome. Very few Saccharomyces genes have introns, presumably due to the selective pressure for efficient growth, perhaps intensified by its centuries or millenia of domestication. Yet nearly all of the yeast genes for ribosomal proteins have a single intron (for review, see Warner et al. 1986). The recent proposal that ribosomal protein L32 can regulate the splicing of the transcript of its own gene (Dabeva et al. 1986) provides a reason why ribosomal protein genes may have retained their introns. Some years ago temperature-sensitive $(t s)$ mutants were identified, which, at the restrictive temperature, appeared to block the synthesis of ribosomes rather specifically (Hartwell et al. 1970) due to a depletion of mRNA for ribosomal proteins (Warner and Gorenstein 1978). It subsequently became clear that the de- 
fect is in the ability to splice out any intron (Rosbash et al. 1981). The apparent ribosome specificity of the mutants is due to the preponderance of introns in the genes for ribosomal proteins. More than $5 \%$ of the $t s$ mutations isolated on rich media led to defects in splicing, suggesting that they represent a large number of genes. The original group of 23 fell into 10 complementation groups, termed $R N A 2-R N A 11$, with statistics implying that there are many more (Hartwell et al. 1970). They are distinct from $R N A 1$, a gene that is essential for the splicing of tRNA precursors but not mRNA precursors (Hopper and Banks 1978).

Several of the RNA genes have been cloned (Last et al. 1984; Lee et al. 1984; Soltyk et al. 1984). They are essential for growth, but they do not themselves contain introns. Using immunological techniques, Last and Woolford (1986) have shown that the RNA2 product is about $100,000 \mathrm{~m} . \mathrm{w}$. and the RNA3 product is about 55,000 . They are both found in nuclei, as would be expected for participants in splicing.

The value of the conditional RNA mutants for understanding the biochemistry of splicing has become apparent from the recent finding that the extracts derived from several of the $t s$ mutants are heat labile for the in vitro splicing reaction. Furthermore, pairs of mutants that complement in vivo yield extracts that complement in vitro (Lustig et al. 1986). In this issue of Genes \& Development, Lin et al. (1987) report that heat-treated extracts of a ts mutant in RNA2 can form normal spliceosomes whose substrate is unspliced, but equivalent extracts of $t s$ mutants in $R N A 3,-4,-5,-7,-8$, or -11 cannot. Furthermore, the spliceosomes formed in a heat-treated extract of cells carrying the temperature-sensitive allele of $R N A 2$ can be isolated. When they are added to a heattreated extract of a mutant in $R N A 3,-4,-5,-7,-8$, or -11 , the substrate RNA molecule they contain is spliced efficiently and rapidly, and in preference to an alternative substrate added simultaneously. This result implies either that the products of $R N A 3,-4,-5,-7,-8$, or -11 genes are in the spliceosome or that they have carried out their function before the spliceosome was isolated from the RNA2-defective extract. Experiments using a strain which overproduces the RNA2 gene product suggest that some additional factor is also essential to complete the splicing of a substrate molecule already assembled into a spliceosome.

Clearly the next step, already underway /Cheng and Abelson 1986), is to fractionate the extract used to complement the spliceosome from a ts mutant extract. Eventually, the system can be utilized to purify each of the complementing gene products, an approach that has had great utility in solving complex biochemical pathways, such as DNA synthesis. In addition, cloning the gene involved in mRNA processing provides the basis for preparing immunological reagents to probe the spliceosome (Last and Woolford 1986). When one is able to obtain reasonably pure spliceosomes, it ought to be possible to apply the immunoelectron microscopy techniques that have been so powerful in establishing the structure of the ribosome (Oakes et al. 1986). Finally, with all those mutants one can foresee the geneticists isolating suppressors indefinitely, bringing the system full circle, with mutants in genes for some of the components suppressing $t s$ mutants in the genes of others. Indeed, Pearson et al. (1982) have identified a mutant that is a dominant suppressor of several ts RNA mutants, but it has not yet been analyzed at the molecular level. R.L. Last and J.L. Woolford (pers. comm.) have isolated a mutant that suppresses the $t s$ RNA2 mutation, but not a deletion of RNA2.

Recently attention has turned to the fission yeast Schizosaccharomyces pombe, which combines the genetic manipulability of Saccharomyces with a splicing system more characteristic of higher organisms. Introns are far more prevalent (Hindley and Phear 1984). Its U2 RNA is also identical in size to that of mammals (Ares 1986; Brennwald, Porter, and Wise, pers. comm.). At least one mammalian gene, SV40 T antigen, can be spliced correctly (Kaufer et al. 1985) in S. pombe.

It is tempting to think of the splicing apparatus as analogous to the translation apparatus, each established early in evolution to bring about the correct juxtaposition of gene fragments or sequences. Each is composed of a complex particle containing RNA and protein, as well as auxiliary soluble factors. A contrast, however, is that the spliceosome appears to disassemble substantially between operations, whereas the ribosome remains largely intact. Perhaps this is why temperaturesensitive mutants for spliceosome function are found so readily while such mutants in ribosome function (as opposed to assembly) are rare. Indeed, none were found in the screen that generated 23 mutants impaired in splicing (Hartwell and McLaughlin 1968). In any case, the availability of the mutants in RNA2-RNA10 and the likelihood of many more, the subsequent isolation of the genes themselves with their promise of gene disruption experiments and overexpressed gene products available for biochemical utilization, and the variety of genetic manipulations available in yeast, all suggest that Saccharomyces will make major contributions to our understanding of the splicing apparatus and its operation in the next few years.

\section{Jonathan R. Warner}

Departments of Cell Biology and Biochemistry

Albert Einstein College of Medicine

Bronx, New York 10461 USA

\section{References}

Ares, M., Jr. 1986. U2 RNA from yeast is unexpectedly large and contains homology to vertebrate U4, U5, and U6 small nuclear RNAs. Cell 47: 49-59.

Brody, E. and J. Abelson. 1985. The "spliceosome:" Yeast premessenger RNA associated with a 405 particle in a splicingdependent reaction. Science 228: 963-967.

Cech, T.R. and B.L. Bass. 1986. Biological catalysis by RNA. Annu. Rev. Biochem. 55: 599-630.

Cheng, S.-C. and J. Abelson. 1986. Fractionation and characterization of a yeast mRNA splicing extract. Proc. Natl. Acad. Sci. 83: 2387-2391. 
Dabeva, M.D., M.A. Post-Beittenmiller, and J.R. Warner. 1986. Autogenous regulation of splicing of the transcript of a yeast ribosomal protein gene. Proc. Natl. Acad. Sci. 83: 58545857.

Domdey, H., B. Apostol, R.-J. Lin, A. Newman, E. Brody, and J. Abelson. 1984. Lariat structures are in vivo intermediates in yeast pre-mRNA splicing. Cell 39: 611-621.

Green, M.R. 1986. Pre-mRNA splicing. Annu. Rev. Genet. 20: $671-708$.

Guthrie, C., N. Riedel, R. Parker, H. Swerdlow, and B. Patterson. 1986. Genetic analyses of snRNAs and RNA processing in yeast. In Yeast cell biology, pp. 301-321. A.R. Liss, New York.

Hartwell, L.H. and C.S. McLaughlin. 1968. Temperature-sensitive mutants of yeast exhibiting a rapid inhibition of protein synthesis. I. Bacteriol. 96: 1664.

Hartwell, L., C. McLaughlin, and J.R. Warner. 1970. Identification of ten genes that control ribosome formation in yeast. Mol. Gen. Genet. 109: 42-56.

Hindley, J. and G.A. Phear. 1984. Sequence of the cell division gene CDC2 from Schizosaccharomyces pombe; patterns of splicing and homology to protein kinases. Gene 31: 129134.

Hopper, A.K. and F. Banks. 1978. A yeast mutant which accumulates precursor tRNAs. Cell 14: 211-219.

Kaufer, N.F., V. Simanis, and P. Nurse. 1985. Fission yeast Schizosaccharomyces pombe correctly excises a mammalian RNA transcript intervening sequence. Nature 318: 7880.

Last, R.L., I.B. Stavenhagen, and J.L. Woolford, Jr. 1984. Isolation and characterization of the RNA2, RNA3, and RNA11 genes of Saccharomyces cerevisiae. Mol. Cell. Biol. 4: 2396-2405.

Last, R.L. and J.L. Woolford, Ir. 1986. Identification and nuclear localization of yeast pre-messenger RNA processing components: RNA2 and RNA3 proteins. $J$. Cell Biol. 103: 21032112.

Lee, M.G., R.A. Young, and J.D. Beggs. 1984. Cloning of the RNA2 gene of Saccharomyces cerevisiae. EMBO I. 3: 28252830.

Lin, R.-J., A.J. Lustig, and J. Abelson. 1987. Splicing of yeast nuclear pre-mRNA in vitro requires functional 40 S spliceosome and several extrinsic factors. Genes Dev. 1: 17-18.

Lustig, A.J., R.-J. Lin, and J. Abelson. 1986. The yeast RNA gene products are essential for mRNA splicing in vitro. Cell 47: 953-963.
Miller, A.M. 1984. The yeast MATal contains two introns. EMBO I. 3: 1061-1065.

Oakes, M., E. Henderson, A. Scheinman, M. Clark, and J.A. Lake. 1986. Ribosome structure, function, and evolution: Mapping ribosomal RNA, proteins, and functional sites in three dimensions. In Structure, function, and genetics of ribosomes (ed. B. Hardesty and G. Kramer), pp. 47-67. Springer-Verlag, New York.

Padgett, R.A., P.J. Grabowski, M.M. Konarska, S. Seiler, and P.A. Sharp. 1986. Splicing of messenger RNA precursors. Annu. Rev. Biochem. 55: 1119-1150.

Pearson, N.J., P.C. Thornburn, and J.E. Haber. 1982. A suppressor of temperature sensitive ma mutations that affect general and specific messenger RNA processing in yeast. Mol. Cell. Biol. 2: 571-577.

Pikielny, C.W. and M. Rosbash. 1986. Specific small nuclear RNAs are associated with yeast spliceosomes. Cell 45: 869877.

Pikielny, C.W., B.C. Rymond, and M. Rosbash. 1986. Electrophoresis of ribonucleoproteins reveals an ordered assembly pathway of yeast splicing complexes. Nature 324: 341-345.

Riedel, N., I.A. Wise, H. Swerdlow, A. Mak, and C. Guthrie. 1986. Small nuclear RNAs from Saccharomyces cerevisiae: Unexpected diversity in abundance, size, and molecular complexity. Proc. Natl. Acad. Sci. 83: 8097-8101.

Rodriquez, J.R., C.W. Pikielny, and M. Rosbash. 1984. In vivo characterization of yeast mRNA processing intermediates. Cell 39: 603-610.

Rosbash, M., P.K.W. Harris, J.L. Woolford, Ir., and J.L. Teem. 1981. The effect of temperature sensitive RNA mutants on the transcription products from cloned ribosomal protein genes of yeast. Cell 24: 679-686.

Rymond, B.-C. and M. Rosbash. 1985. Cleavage of $5^{\prime}$ splice site and lariat formation are independent of $3^{\prime}$ splice site in yeast mRNA splicing. Nature 317: 735-737.

Soltyk, A., M. Tropak, and J.D. Friesen. 1984. Isolation and characterization of the $R N A 2^{+}, R N A 4^{+}$, and $R N A 11^{+}$genes of Saccharomyces cerevisiae. I. Bacteriol. 160: 1093-1100.

Warner, J.R., E.A. Elion, M.D. Dabeva, and W.F. Schwindinger. 1986. The ribosomal genes of yeast and their regulation. In Structure, function, and genetics of ribosomes led. B. Hardesty and G. Kramer|, pp. 719-732. Springer-Verlag, New York.

Warner, I.R. and C. Gorenstein. 1978. Yeast has a true stringent response. Nature 275: 338-339.

\section{Studying plant development: an alternative to "spray and pray"}

In this issue, Klee et al. (1987) report the endogenous overproduction of the plant hormone auxin, and the effect it has on plant development. This is a major advance over previous studies, all of which depend on exogenous hormone application, or disrupting the integrity of the plant. The in vivo manipulation of endogenous hormone levels makes it now possible to characterize the pleiotropic effects of hormones throughout the life cycle of the plant. Whereas animal cells become committed to a specific developmental program at an early stage, plant cells retain an amazing degree of flexibility (Wareing and Phillips 1981). Even differentiated cells can be repro- 


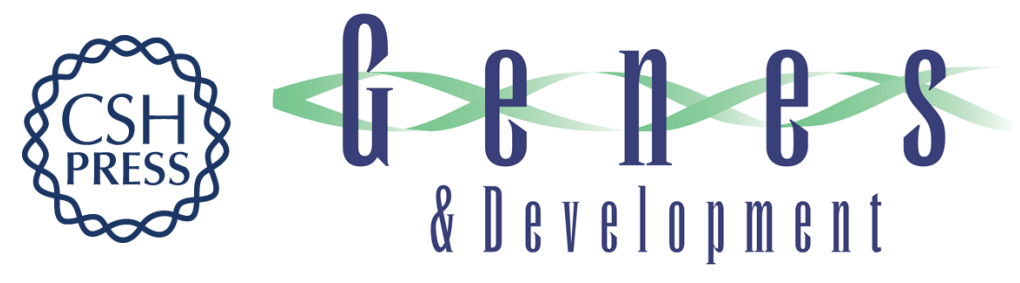

\section{Applying genetics to the splicing problem}

Jonathan R. Warner

Genes Dev. 1987, 1:

Access the most recent version at doi:10.1101/gad.1.1.1

References This article cites 28 articles, 9 of which can be accessed free at: http://genesdev.cshlp.org/content/1/1/1.full.html\#ref-list-1

License

Email Alerting Receive free email alerts when new articles cite this article - sign up in the box at the top Service right corner of the article or click here.

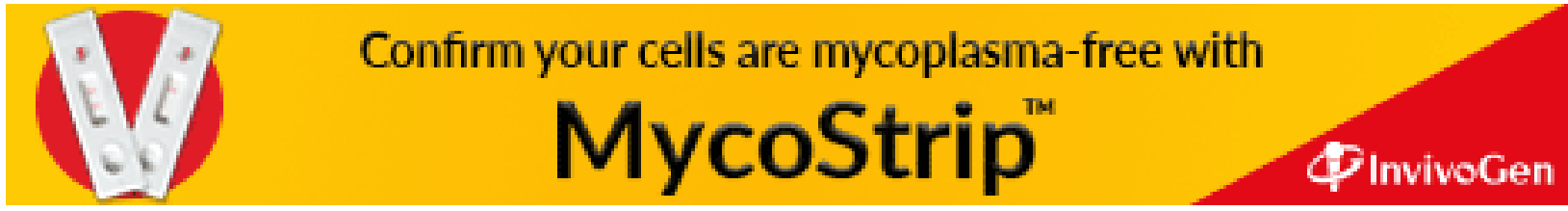

